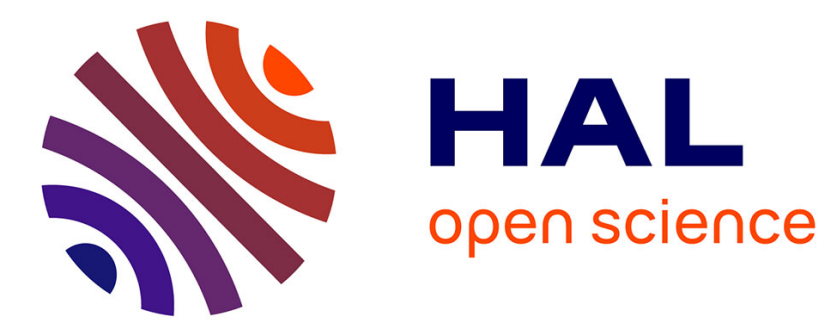

\title{
Le mouvement abolitionniste français dans l'après-guerre.
}

\author{
Lilian Mathieu
}

\section{To cite this version:}

Lilian Mathieu. Le mouvement abolitionniste français dans l'après-guerre. : Recompositions et reconversions de la croisade contre la prostitution réglementée. Déviance et Société, 2016, 40 (1), pp.79-100. halshs-02057153

\section{HAL Id: halshs-02057153 \\ https://shs.hal.science/halshs-02057153}

Submitted on 5 Mar 2019

HAL is a multi-disciplinary open access archive for the deposit and dissemination of scientific research documents, whether they are published or not. The documents may come from teaching and research institutions in France or abroad, or from public or private research centers.
L'archive ouverte pluridisciplinaire HAL, est destinée au dépôt et à la diffusion de documents scientifiques de niveau recherche, publiés ou non, émanant des établissements d'enseignement et de recherche français ou étrangers, des laboratoires publics ou privés. 


\section{Lilian Mathieu}

Centre Max Weber-CNRS

ENS de Lyon

15 parvis Descartes

69007 Lyon

Tel : 0437376437

lilian.mathieu@ens-lyon.fr

\section{Le mouvement abolitionniste français dans l'après-guerre : Recompositions et reconversions de la croisade contre la prostitution réglementée}

Relativement mal connue, la période 1946-1960 est importante dans l'histoire du mouvement français pour l'abolition de la réglementation de la prostitution. Cette séquence ne donne pas seulement lieu à une lutte pour la suppression, après la fermeture des maisons closes, du fichier médico-social obligatoire des prostituées. Elle est surtout celle d'un effacement des courants abolitionnistes historiques, liés à la bourgeoisie protestante et dont le discours associe traite des blanches et réglementarisme, au profit d'une mouvance nouvelle, issue du catholicisme social et promotrice d'une approche davantage sociale du phénomène prostitutionnel. C'est à cette mouvance, principalement incarnée par le Nid, que la conversion de la France à l'abolitionnisme va donner les moyens de prospérer à partir de 1960.

Prostitution - abolitionnisme - réglementarisme - catholicisme social - réinsertion

Though under-studied, the years 1946-1960 are important in the history of the French movement for the abolition of the regulation of prostitution. The period has not only witnessed a struggle, after the ban on brothels, for the suppression of the compulsory medical and social registration of prostitutes. It is mainly the period of a weakening of the main historical abolitionist currents, that were linked to the Protestant bourgeoisie and whose rhetoric associated regulation with white slave traffic, for the benefit of a new movement coming from social Catholicism and promoting a more social approach of prostitution. The main expression of this movement is le Nid (the Nest); it was given the means to prosper after the complete conversion of France to abolitionism in 1960.

Prostitution - abolitionism - regulation - social Catholicism - rehabilitation

L'après-Seconde Guerre mondiale est une période importante, mais relativement mal connue, dans l'histoire de la croisade pour l'abolition de la réglementation de la prostitution ${ }^{1}$. Attentifs à son émergence et à son développement, les historiens cessent de s'y intéresser après l'épisode de Vichy, tandis que l'étude sociologique des

\footnotetext{
${ }^{1}$ Sur la notion de croisade morale, voir Mathieu (2005).
} 
controverses contemporaines montre peu d'intérêt pour un contexte législatif révolu. Les années 1946-1960 souffrent spécialement de leur situation intermédiaire entre un réglementarisme atteint dans son cœur par l'interdiction des maisons closes mais qui survit via l'inscription obligatoire des prostituées sur un fichier médico-social (lois des 13 et 24 avril 1946), et la conversion complète de la France à l'abolitionnisme par la ratification de la Convention de l'ONU pour la répression de la traite des êtres humains et de l'exploitation de la prostitution d'autrui (ordonnance du 28 juillet 1960), qui impose d' "abroger ou abolir toute loi, tout règlement ou toute pratique administrative selon lesquelles les personnes qui se livrent ou sont soupçonnées de se livrer à la prostitution doivent se faire inscrire sur des registres spéciaux, posséder des papiers spéciaux, ou se conformer à des conditions exceptionnelles de surveillance ou de déclaration ».

La séquence 1946-1960 n'est pas seulement, pour les abolitionnistes français, celle qui précède leur victoire définitive sur le réglementarisme. Elle est également une période de forte recomposition de leur mouvement - de son recrutement, de ses références, de ses positions et de ses activités - au point qu'on puisse considérer qu'elle est le moment de la fin d'un certain abolitionnisme et de sa transfiguration sous de nouveaux traits. C'est à ces transformations que l'on s'attachera dans cet article à vocation principalement documentaire, à partir de l'étude des deux principales composantes du mouvement: le Cartel d'action morale et sociale, d'une part, héritier direct de la Ligue française pour le relèvement de la moralité publique, et le Nid, d'autre part, officiellement créé en 1946 dans la mouvance de l'Action catholique ouvrière. Les publications trimestrielles de ces deux organisations - Rénovation que le Cartel lance en 1947, et Moissons nouvelles publié par le Nid à partir de 1951 - ont pour ce faire été systématiquement dépouillées ${ }^{2}$. S'y ajoutent les écrits des principaux «intellectuels organiques » de l'abolitionnisme français ainsi que les archives de l'Union française contre le trafic des femmes ${ }^{3}$.

\section{L'abolitionnisme français en son contexte}

Les premières années du mouvement pour l'abolition de la réglementation de la prostitution sont aujourd'hui bien documentées (Corbin, 1978 ; Walkowitz, 1980). On connaît avec précision le rôle décisif joué dans l'Angleterre des années 1860 par la classe moyenne protestante, et plus spécifiquement par ses composantes féminines et non-conformistes dont Josephine Butler incarne la figure la plus hérö̈que (Käppeli, 1990 ; Regard, 2013). On sait également comment ce mouvement est parvenu à s'internationaliser dès la fin des années 1870 en s'appuyant à la fois sur les réseaux féminins de la bourgeoisie protestante - suisse et française, spécialement (Corbin, 1978 ; Käppeli, 1990 ; Rochefort, 2008) —, sur un usage émotionnel de la thématique de la «traite des blanches » (Chaumont \& Machiels, 2009) et sur un équilibre instable entre positions progressistes et féministes, d'une part, et courants puritains promoteurs de la décence publique et de la continence préconjugale d'autre part (Bland, 1992). On connaît également les conditions de l'appropriation institutionnelle de la cause

\footnotetext{
2 Rénovation cesse de paraître en 1970 ; Moissons nouvelles (abrégé en MS dans les références) devient en 1968 Femmes et mondes puis, en 1989, Prostitution et société, son titre actuel. Les deux revues ne comptant qu'un nombre limité de pages (simple recto-verso dans le cas de Rénovation et des premiers numéros de $M S$ ) on n'a indiqué la pagination que pour les numéros spéciaux de $M S$ parus sous forme de brochures.

${ }^{3}$ Fonds Marcelle Legrand-Falco déposé au Musée social de Paris.
} 
abolitionniste à compter du début du $\mathrm{XX}^{\mathrm{e}}$ siècle, lorsque la lutte contre la traite des blanches a commencé à faire l'objet de conventions d'abord entre États puis sous les auspices de la Société des nations (Chaumont, 2009; Limoncelli, 2010).

Cause politiquement et scientifiquement hérétique avant 1914, l'abolition de la réglementation de la prostitution gagne en légitimité pendant l'entre-deux guerres, à mesure que s'y rallient des médecins désormais dubitatifs quant au bien fondé prophylactique du contrôle sanitaire des seules prostituées (Corbin ; 1978 ; DuffulerVialle, 2014). Elle peut en outre s'appuyer sur les précédents exemplaires de pays (telle la Hollande à partir de 1911) ou de villes (comme Grenoble, Strasbourg ou Colmar) qui ont mis fin à la réglementation sans subir de catastrophe sanitaire ou morale notable. Cette légitimation permet à un mouvement jusque-là principalement animé par des femmes de la bourgeoisie protestante de s'ouvrir aux fractions dominantes d'autres couches sociales. Mise en place en 1926, l'Union temporaire contre la prostitution réglementée et la traite des êtres humains rassemble un éventail large d'organisations en majorité confessionnelles mais également laïques. On peut interpréter comme une attestation d'œcuménisme en même temps que comme une allégeance à la hiérarchie des confessions la composition de son comité d'honneur, présidé par l'archevêque de Paris sous l'autorité duquel siègent le président de la Fédération protestante, le Grand rabbin de Paris, le Président de la Ligue des droits de l'homme, des professeurs de la Sorbonne et du Collège de France, ainsi que des professeurs de médecine.

Conformément à la signification que possède le terme depuis son origine, ce que vise l'abolitionnisme est l'abolition de la réglementation et non de la prostitution ellemême - « nous ne sommes pas assez naïfs pour nourrir une telle ambition » déclare l'universitaire strasbourgeois Paul Gemälhing, président de l'Union temporaire, dans son bulletin annuel de $1936\left(n^{\circ} 6,18\right)$. Le contrôle sanitaire obligatoire des prostituées est dénoncé comme inefficace et, pire encore, comme donnant une fausse impression de sécurité qui encourage au vice et par suite à la propagation des maladies. Ayant pignon sur rue, le bordel incite les hommes - et spécialement les jeunes gens - à la débauche en leur facilitant les moyens de satisfaire leurs désirs. Mais c'est bien entendu sur les prostituées elles-mêmes qu'il exerce ses effets les plus délétères: "Réduites à une dégradation totale par une alcoolisation systématique et une démoralisante oisiveté, (...) elles devront, à toute réquisition, se soumettre à la bestialité de tous ceux qui pourront se présenter, hommes de toutes races, individus en état d'ivresse ou atteints de maladies répugnantes, débauchés, cherchant à satisfaire là les pires dépravations des sens » (Gemälhing, 1933, 43). Un projet de loi de Paul Sellier, ministre de la Santé du gouvernement du Front populaire, visant à interdire les maisons closes soulève les espoirs des abolitionnistes mais se heurte à l'opposition de l'Amicale des maîtres d'hôtels meublés de France et des colonies, regroupant les tenanciers de bordels, qui parvient à empêcher qu'il soit examiné par le Sénat.

L'arrivée au pouvoir de Pétain marque une déroute des abolitionnistes. Le régime de Vichy voit dans les conduites prostitutionnelles un risque de dégénérescence qu'il tente de conjurer par un réglementarisme policier associé à une politique antivénérienne des plus strictes. La crainte d'une diffusion incontrôlable des pratiques dégénérées, la répression de l'«esprit de jouissance», une conception rigide et naturalisée de la division entre les sexes, donnent une nouvelle vigueur à la volonté de contention de la prostitution dans les maisons closes (Olivier, 2005). A contrario, la Libération ouvre aux abolitionnistes une fenêtre d'opportunité dont ils savent se saisir. Dans plusieurs villes, la collaboration des tenanciers avec les Allemands les disqualifie suffisamment pour que personne n'ose défendre leur activité, ainsi que le relate le 
pasteur Lasserre dans son récit de la manière dont les "maisons furent fermées »: «Avec quelle obséquiosité n'avaient-ils pas réservé leurs maisons - et nos filles - aux occupants! Pour la première fois, leur position était relativement fragile : ne fallait-il pas en profiter rapidement, avant qu'ils n'aient de nouveau acheté toutes les complicités habituelles ? » (Lasserre, 1955, 12).

Le récit mythifié de la « fermeture » (attribuant à Marthe Richard un rôle décisif qu'elle n'a pas tenu) oublie que l'Assemblée nationale ayant toujours refusé de débattre d'un sujet aussi indigne que la prostitution, c'est au niveau local que celle-ci a été réglementée. C'est par conséquent à ce niveau, en ciblant les nouvelles municipalités issues de la Résistance, que les abolitionnistes ont dès la fin 1944 fait campagne pour, ville par ville, obtenir la fermeture des bordels. Le récit que livre le pasteur Lasserre de son action dans le département du Nord est riche d'enseignement sur la manière dont a été menée cette campagne. Celui-ci a tout d'abord cherché un soutien auprès des différents courants de la Résistance afin de consolider un front unanime - «Parlèrent (...) quatre délégués municipaux, un CGT, un Front national, un MRP, et un SFIO. Le cinquième, communiste, s'était fait excuser: il avait toujours été très ferme pour soutenir notre cause » (ibid., 17-18) —, s'est appuyé sur les réseaux chrétiens locaux «Un groupe de militants catholiques, jocites ou MPF, (...) faisaient joyeusement équipe avec nous, et nous apportèrent une aide particulièrement utile » (ibid., 25) - et a organisé des débats publics afin de sensibiliser la population à la nécessité de supprimer les maisons closes, en insistant sur le fait que, dans les bordels, des soldats américains noirs pouvaient accéder sexuellement à des Françaises ${ }^{4}$.

Menée localement, la campagne abolitionniste n'en est pas moins coordonnée, au niveau national, par le Cartel d'action morale et sociale qui a assuré un intense lobbying sur le conseil municipal de Paris (ibid., 131) puis sur le Parlement ${ }^{5}$. Après quelques tergiversations, le ministre MRP (Mouvement républicain populaire) de la Santé et de la Population Robert Prigent fait adopter le 13 avril 1946 un texte législatif qui interdit les maisons de tolérance sur l'ensemble du territoire - métropolitain seulement ${ }^{6}$. Parallèlement, cette loi institue, en satisfaisant également les revendications abolitionnistes, le délit de racolage et redéfinit en les aggravant les peines encourues pour proxénétisme (Brunet, 1990). L'enthousiasme des abolitionnistes est cependant douché par l'instauration, par la loi du 24 avril 1946 défendue par le médecin et député socialiste Denis Cordonnier, d'un fichage médico-social des prostituées dont ils continuent à contester le bien fondé en matière de santé publique (Maugère 2009, p. 166). C'est, de leur point de vue, la réinsertion - ou plutôt, dans les termes de l'époque, le reclassement ou le relèvement - des prostituées qui s'impose désormais comme la priorité.

\section{Recompositions organisationnelles}

Le récit qui précède le montre, les abolitionnistes ont joué un rôle décisif dans la décision politique d'interdiction des maisons closes. Reste que leur mouvement a été affecté par la guerre; si la Libération ouvre une opportunité de remobilisation, elle

\footnotetext{
${ }^{4}$ Le fait est signalé à plusieurs reprises dans son ouvrage (Lasserre 1955, p. 19 et 26-27). L'auteur s'indigne un peu plus loin que «c'est dans ces maisons, où l'on a osé leur fournir des femmes blanches, que les Nord-Africains, notamment, ont pris l'habitude de mépriser les femmes de chez nous » (p. 136).

${ }^{5}$ Les archives de Marcelle Legrand-Falco laissent notamment apparaître une intense correspondance avec le député MRP Pierre Dominjon.

${ }^{6}$ Sur le maintien du réglementarisme en Afrique du Nord, cf. Taraud (2003).
} 
implique également une recomposition de ses formes organisationnelles. Les deux associations ici étudiées présentent sur ce plan des situations contrastées : le Cartel d'action morale et sociale est la nouvelle appellation d'une structure préexistante tandis que la création du Nid signale un investissement catholique d'ampleur inédite dans la cause de l'abolition définitive de la réglementation. Pour autant, leurs rapports ne sont pas concurrentiels mais davantage fondés sur un partage des tâches, le Cartel menant une action de sensibilisation de l'opinion et de lobbying politique tandis que le Nid se spécialise dans le secours aux prostituées.

\section{Le Cartel, une œuvre de relèvement moral}

Fondé pendant la guerre, le Cartel d'action morale et sociale contre les fléaux destructeurs de la vie française (appellation complète de ce que l'on désignera désormais comme le Cartel) fusionne à la Libération avec la Ligue française pour le relèvement de la moralité publique, elle-même fondée en 1883 et, avant-guerre, membre active de l'Union temporaire contre la prostitution réglementée et la traite des êtres humains. L'Union temporaire se reconstitue au même moment en tant qu'Union française contre le trafic des femmes et devient la section française de la Fédération abolitionniste internationale (fondée par J. Butler en 1877).

Cartel et Union française sont donc la continuation directe des principales organisations abolitionnistes de l'entre-deux guerre ${ }^{7}$. Elles entretiennent des liens d'autant plus étroits que la multipositionnalité de leurs membres est fréquente. Le président du Conseil national du Cartel est ainsi l'ancien président de l'Union temporaire Paul Gemälhing et plusieurs personnalités présentes avant-guerre au sein de cette dernière siègent au conseil national du Cartel. C'est le cas, par exemple, du $\mathrm{D}^{\mathrm{r}}$ Sicard de Plauzolles, secrétaire général de la Ligue française contre le péril vénérien, et de Marcelle Legrand-Falco, veuve d'un général et infirmière engagée dans le soutien aux prostituées à leur sortie de prison, ancienne secrétaire générale de l'Union temporaire et nouvelle présidente de l'Union française. Dans la continuité de l'histoire de l'abolitionnisme français, on constate parmi les parrains ou animateurs du Cartel la forte présence de noms connus de la bourgeoisie intellectuelle protestante - Gustave et Théodore Monod, André Siegfried (qui accueille le Cartel au Musée social), l'ethnologue et pasteur Jacques Leenardt ou encore le $\mathrm{D}^{\mathrm{r}}$ Édouard Rist (membre de l'Académie de médecine et frère de l'économiste Charles Rist). On constate également - là encore dans la continuité d'une lutte d'expertise contre les tenants du contrôle sanitaire des prostituées - la surreprésentation de médecins, souvent professeurs de faculté.

Les organisations parties prenantes du Cartel se signalent quant à elles par leur attention pour l'état sanitaire et moral de la population, et tout spécialement de la jeunesse et de la famille : Ligue nationale contre l'alcoolisme, Alliance nationale contre la dépopulation, Ligue française contre le péril vénérien, École des parents, Ligue féminine d'action catholique, Union chrétienne des jeunes gens, Mouvement populaire des familles (MPF), Scoutisme français ou encore Jeunesse ouvrière chrétienne (JOC). Poursuivant la tradition d'œcuménisme de l'abolitionnisme français, le comité d'honneur de l'Union française accueille quant à lui des personnalités d'horizons politiques ou religieux divers (fréquemment liés à la Résistance), à l'instar de la communiste Jeannette Vermeersch, du gaulliste Maurice Schumann, du MRP Léo Hamon, $\mathrm{du}$ radical-socialiste Justin Godart, du chanoine Viollet (ces deux derniers également

\footnotetext{
${ }^{7}$ Le Cartel ayant manifestement connu une activité publique beaucoup plus dense que l'Union française (dont, au regard des archives de M. Legrand-Falco, l'action paraît plus réduite qu'avant-guerre) tout en partageant des positions similaires, c'est à lui que l'on a donné la priorité dans l'analyse.
} 
membres du Cartel), du cardinal Jules Saliège, du pasteur Marc Boegner ou du rabbin Julien Weill.

Le Cartel est animé par l'ingénieur Daniel Parker, fervent protestant auteur de plusieurs opuscules tels que Les Trafiquants de femmes (1938), Comment lutter contre la littérature pornographique (1939), Puissance et responsabilité du film (1945) ou Le Respect de la dignité humaine et l'Immoralité dans les lieux de travail (1946), pour la plupart édités par le Cartel. Car quoique tenant la prostitution pour un de ses principaux champs de bataille, l'association se préoccupe plus largement de lutter contre les multiples périls qui menacent la santé physique et morale de la population française. L'éditorial du premier numéro de Rénovation (juin 1947), signé par Gemälhing, se réclame de Giraudoux pour poser le "problème du nombre et de la qualité des Français ». "Ce n'est pas avec des alcooliques et des avariés que nous reconstituerons la France », affirme le professeur de droit, qui dresse la liste des chantiers que le Cartel souhaite voir ouvrir : "la destruction des taudis, une lutte résolue contre l'alcoolisme, l'avortement et le proxénétisme, le relèvement et le reclassement des prostituées, la préservation et la rééducation de l'enfance déficiente et délinquante, la préservation de la jeunesse contre l'immoralité de la rue, du cinéma, la sauvegarde de la dignité des jeunes travailleurs ». À la fin des années 1940, le Cartel se fait connaître par ses actions judiciaires pour faire interdire les romans J'irai cracher sur vos tombes de Boris Vian et Tropique du cancer d'Henry Miller, et joue de son influence dans l'élaboration de la loi de 1949 sur les publications destinées à la jeunesse. Le cinéma, spécialement, est systématiquement dénoncé dans Rénovation comme une "école du vice et du crime » $\left(\mathrm{n}^{\circ} 10,1950\right)$. Les maires sont interpellés $\left(\mathrm{n}^{\circ} 39,1959\right)$ pour qu'ils usent de leurs pouvoirs en matière de surveillance des spectacles potentiellement immoraux (théâtres, music-halls, kermesses, foires, bals et dancings) et de suppression de l'affichage licencieux. Opposant résolu au divorce, à la contraception et à l'avortement, le Cartel affirme que le slogan " maternité voulue, maternité heureuse » est « expérimentalement faux » et dénonce un « climat de facilité sexuelle qui risquerait de hâter la dégradation de notre civilisation $»\left(\mathrm{n}^{\circ} 44-45,1961\right)$.

S'agissant plus spécifiquement de la prostitution, le Cartel s'appuie sur l'expertise de médecins pour réfuter que la fermeture des «maisons » puisse avoir une influence sur la propagation des maladies vénériennes. Le danger, selon eux, réside prioritairement - outre "l'arrivée en France de travailleurs étrangers » - dans le " relâchement des mœurs sexuelles, conduisant à une prostitution plus difficilement définissable » $\left(n^{\circ} 1,1947\right)$. Les anciennes angoisses devant la prostitution insaisissable des «clandestines» (Corbin, 1978) ne sont pas mortes et le maintien d'un fichage obligatoire apparaît voué à l'échec. Discours typique d'entrepreneur de morale, celui que le Cartel tient sur les prostituées présente lui aussi une "coloration humanitaire marquée » (Becker, 1985, 172). Rénovation s'indigne dans son $n^{\circ} 6$ que (1949) que, trois ans après l'interdiction des maisons closes, « les femmes continuent à être traitées (...) par les agents des mœurs, par quelques médecins cyniques et profiteurs, par l'opinion publique elle-même, comme un bétail humain, dont on ne peut se rendre maître que par la rafle, la "visite sanitaire", l'incarcération dans un hôpital-prison ». Le Cartel milite à l'inverse pour la suppression de la police des mœurs, la disparition de toutes les mesures d'exception et pour la prévention et le reclassement de «tant de malheureuses qui cherchent à s'évader de la tyrannie de l'habitude et de celle de leur souteneur» (ibid.). C'est ce dernier créneau, à dimension prioritairement sociale, qu'investit au même moment une nouvelle association, le Nid. 


\section{L'expérience sociale du Nid}

L'investissement du terrain social par le mouvement abolitionniste n'est toutefois pas totalement nouveau. Ville pionnière (les maisons closes y ont été interdites dès 1930), Grenoble a accueilli L'Abri dauphinois destiné dès 1932 aux anciennes prostituées. S'y sont ajoutés à la Libération La Cordée à Besançon, L'Abri languedocien à Montpellier ainsi qu'à Paris La Bienvenue et le foyer Josephine Butler - cela sans compter les foyers du Bon Pasteur, tenus par des religieuses et destinés aux «filles mères » et «filles perdues », qui hébergent leur lot d'ex, mais aussi futures, prostituées (Blanchard, 2008).

L'initiative la plus significative, et appelée à devenir exemplaire, est cependant le Nid. Celui-ci, selon l'histoire officielle, résulte de la rencontre entre un prêtre breton, André-Marie Talvas, et Germaine Campion, une prostituée alcoolique revenue dans sa région d'origine pour y mourir mais à qui il redonne goût à la vie : "L'abbé Talvas, vicaire de Parramé en Bretagne, eut ce mérite de comprendre une personne, ivrognesse et prostituée, et la témérité d'ajouter malgré l'étonnement de tous : "ne craignez pas, je ne vous abandonnerai jamais". Le Nid commençait en germe » (Séguier, 1954, 62). Talvas et Campion commencent alors à animer dans un manoir de la région malouine un lieu d'accueil pour prostituées et alcooliques. Nommé en 1943 aumônier national adjoint de la Ligue ouvrière chrétienne (qui précède l'Action catholique ouvrière), Talvas s'installe à Paris où il poursuit et développe son accueil de prostituées en détresse, d'abord dans un appartement puis dans un pavillon de Fontenay-aux-Roses. L'investissement de nouveaux membres issus de la JOC permet la formalisation d'un groupe de laïcs désigné à partir de 1946 comme l'« Équipe du Nid ». C'est la même année qu'est officiellement fondée l'association «Le Nid», rapidement devenue Amicale du Nid, et que l'archevêque de Paris, le cardinal Suhard, la reconnaît comme une mission d'Église.

Le Nid s'implante au début des années 1950 à Clichy, où il ouvre un foyer en mesure d'accueillir une cinquantaine de pensionnaires. Un appel lancé en février 1946 dans Témoignage chrétien a permis de réunir de premiers fonds. Les années suivantes, le budget du Nid est pour moitié abondé par des subventions du ministère de la Santé et de la Population, le reste provenant de dons (désignés comme "offrandes ») ainsi que, pour une faible part, des produits de l'atelier (réfection de matelas, confection de couvrepieds et d'édredons) où les ex-prostituées peuvent trouver un premier emploi. L'organisme est de fait en position de dépendance à l'égard des pouvoirs publics, qui sont en mesure d'orienter son activité par leur politique de financement. C'est le cas en 1954, lorsqu'un décret donne la priorité à la prévention auprès des femmes « en danger de prostitution » plutôt qu'au reclassement de celles déjà sur le trottoir. Le Nid doit alors prendre le statut de centre d'hébergement, ce qui lui impose une réduction des durées de séjour selon lui incompatible avec les exigences de rééducation de femmes considérées comme «de grandes malades» parmi lesquelles une moitié seraient des « débiles mentales » $\left(M S \mathrm{n}^{\circ} 20,1956\right)$. Un appel aux dons ${ }^{8}$ permet d'ouvrir en 1953 un centre maternel destiné aux jeunes mères que le foyer ne peut accueillir mais que leur grossesse a incitées à quitter le trottoir. Les années suivantes, le Nid se développe en ouvrant de nouveaux foyers à Épinay, Calais puis Marseille (où le bâtiment est loué aux sœurs Saint-Vincent-de-Paul), Toulon, Bordeaux ou encore Lyon.

\footnotetext{
${ }^{8}$ Les listes de donateurs publiées dans les premiers numéros de $M S$ laissent apparaître une forte proportion d'ecclésiastiques de toute la France, ce qui indique que l'expérience du Nid a été dès ses premières années amplement relayée dans les milieux catholiques.
} 
Dirigé par Talvas, le Nid est composé d'« équipières » aussi désignées comme des missionnaires vouées - selon la devise de la JOC « Nous referons chrétiens nos frères » (Pelletier 2012) - à apporter aux prostituées le secours du message du Christ. Celles-ci sont des laïques consacrées, vivant dans le monde leur engagement à la cause évangélique qu'elles refusent de concevoir comme un célibat: "La missionnaire consacrée (...) est l'épouse de l'Époux par excellence, notre Seigneur Jésus-Christ. Avec lui, elle s'épanouit et se donne aux autres » $\left(M S \mathrm{n}^{\circ} 23,1957,73\right)$. Des équipes masculines sont constituées parallèlement, afin de "consacrer leur vie au relèvement et au reclassement des souteneurs » ainsi qu'au sauvetage des "pédérastes » — " enfants, adolescents, grands jeunes gens et adultes qui (...) sont victimes de cette "maladie", fruit d'une éducation faussée et de mœurs païennes »-, des alcooliques et, plus globalement, d'intervenir dans tous les milieux où «tant de jeunes et d'adultes connaissent des conditions de vie contraires à leur dignité et à leur destinée de fils de Dieu » $\left(M S \mathrm{n}^{\circ} 27\right.$, 1959, 52-53). L'évocation de ce volet d'action spécifiquement masculin s'estompe cependant de la revue du Nid à mesure que les équipes s'ouvrent à la mixité.

Cartel et Nid investissent des créneaux distincts et, sur la période considérée, leurs relations ne semblent par conséquent guère empreintes de concurrence. Le premier se préoccupe avant tout «du contenu des lois» et, jugeant que «celles qui existent ne lui donnent pas satisfaction parce qu'il subsiste telle ou telle forme de mal qui le choque profondément» (Becker, 1985, 171), endosse prioritairement le rôle classique de l'entrepreneur de morale. Il se fait ponctuellement l'écho louangeur des activités du second ("Comment on les sauve : l'exemple du "Nid" », $\mathrm{n}^{\circ} 25,1954$ ), lequel, centré sur sa mission sociale, n'évoque qu'épisodiquement, mais toujours positivement, le travail de pression du Cartel. Il n'est pas pour autant fermé à ses positions puisque Moissons nouvelles accueille régulièrement les articles d'une animatrice du Cartel, l'enquêtrice sociale Odette Philippon. Celle-ci y développe notamment les grandes lignes d'un " plan d'action concret contre l'immoralité » stigmatisant la presse féminine, les «films-crimino-sexuels » ou encore la «propagande anticonceptionnelle » $\left(n^{\circ} 6,1953\right)$. De même la revue du Nid se fait-elle l'écho dans son $n^{\circ} 22$ (1957) de la fondation, par le membre du Cartel et de l'Union française Jean Scelles, des Équipes d'action contre la traite des femmes et des enfants, destinées à militer pour un alourdissement des peines prononcées contre les souteneurs. Ancien parlementaire MRP, passé par le Sillon et la Jeune République de Marc Sangnier ainsi que par la Résistance lorsqu'il résidait en Algérie, Scelles a lancé fin 1956 la nouvelle organisation à l'occasion d'un meeting à la salle Wagram sur «La traite des blanches et l'opinion publique ». Y sont notamment intervenus la députée MRP Francine Lefebvre et l'ancien ministre et député UDSR (Union démocratique et socialiste de la Résistance) Claudius Petit (l'abbé Pierre, lui aussi ancien parlementaire MRP, ayant adressé un message de soutien). De sorte que les recompositions de l'abolitionnisme français sur la période considérée ne prennent pas seulement la forme d'une création de nouvelles organisations investissant des créneaux distincts. Elles assistent aussi à un investissement d'ampleur inédite de courants catholiques (ceux de la JOC, du MRP et du Sillon) au sein d'une mouvance antérieurement dominée par les protestants.

\section{Prostitution et prostituées}

Un trait remarquable de l'abolitionnisme est la vision qu'il développe de ce que sont les femmes qui exercent la prostitution. Sans doute parce qu'il se confronte plus 
directement à la réalité prostitutionnelle, c'est le Nid qui livre les formulations les plus systématiques d'une étiologie psychologique à tonalité compassionnelle et doloriste, pointant des carences individuelles comme origines d'une entrée sur le trottoir pensée comme une « déchéance» (MS $\left.\mathrm{n}^{\circ} 51,1964, \mathrm{p} .102\right)$. Moissons nouvelles, dont les pages regorgent de récits et de portraits édifiants, apparaît ainsi comme un dispositif de sensibilisation (Traïni, 2009) destiné à susciter l'apitoiement et l'engagement de son lectorat en se conformant aux exigences de ce que Luc Boltanski (1993) appelle la topique du sentiment.

Est ainsi posé comme déterminant le fait que les «filles » n'aient «jamais été aimées » et qu'elles souffrent d'une « carence affective qui leur donne le triste courage de faire ce métier » $\left(M S \mathrm{n}^{\circ} 19,1956, \mathrm{p} .4\right)$. S'ils ne sont pas ignorés, les «facteurs sociaux (...) ne peuvent agir que comme occasion favorable non comme cause suffisante. Ils viendront frapper avec une force accrue un équilibre psychique déjà branlant » (ibid., p. 7). Le Nid repère parmi les femmes qu'il accueille « un pourcentage assez impressionnant de maladies mentales » et cite un médecin pour qui « la plupart des prostituées doivent être classées (...) parmi les déséquilibrées, les instables, les perverses ou les débiles mentales » $\left(M S \mathrm{n}^{\circ} 10,1954\right)$. Constat similaire chez le magistrat Marcel Sacotte qui repère une "certaine forme de "débilité mentale" » comme «le fondement profond de leur psychologie» chez «la presque totalité des prostituées » (Sacotte, 1959, 18). Le même auteur souligne leur "niveau d'intelligence très médiocre » (ibid.) dont témoigne à ses yeux le faible nombre des titulaires du certificat d'étude, la fréquence de leurs fautes d'orthographes, leur "absence de discernement souvent déconcertant entre le vrai et le faux» (ibid., 19), leurs "facultés de fabulation» « extraordinaires » (ibid., p. 24), leur « imagination déréglée » (ibid., 25) ou encore leur « crédulité qui dépasse tout ce qu'on peut imaginer » (ibid., 26).

Le symptôme le plus immédiat des carences psychologiques des (futures) prostituées est leur naïveté, qui les prédispose à se laisser abuser par de fausses promesses. Le schème, constitutif de la « figure idéale » de la victime de la traite (Jakšić, 2013), est particulièrement sollicité par Philippon qui consacre un ouvrage - Un Grave danger pour la jeunesse du monde - à intimer aux jeunes gens de « ne pas fuir leur famille car tout est dangereux ailleurs » $(1958,43)$. Ainsi informe-t-elle que « les jeunes filles inexpérimentées, les jeunes rurales ne doivent pas s'engager sans d'extrêmes précautions comme serveuses de bars ou d'établissements publics dans les villes, car elles risquent de tomber dans une maison de prostitution camouflée dont l'espèce prolifère actuellement» (ibid. 10). Ces mises en garde sont illustrées par des récits mélodramatiques mettant en scène des jeunes femmes caractérisées par leur crédulité et leur vulnérabilité, tel celui de «l'imprudente Pauline, qui fréquentait les caves existentialistes, que l'on attache dans une chambre d'hôtel, à moitié nue » (ibid., 67).

Le premier numéro de Moissons nouvelles (octobre 1951) s'ouvre ainsi sur "L'histoire de Gilberte », récit de la descente aux enfers (avant rédemption finale) d'une domestique de ferme méprisée par les filles de son âge car " peignée sans goût, le visage bleuâtre marqué d'hérédité alcoolique, les mains grossières sentant l'écurie », soumise aux avances brutales du fils du patron et qui rêve de la ville - où elle finit par s'enfuir et par sombrer dans la prostitution - en lisant la presse du cœur. On y retrouve la plupart des traits structurants des parcours de prostituées que mettent en récit les abolitionnistes : l'opposition entre la campagne arriérée et la ville pleine de périls ${ }^{9}$, celle entre l'innocence féminine et la débauche masculine, celle aussi entre une condition

\footnotetext{
${ }^{9}$ Cet imaginaire de la ville n'est pas sans parenté avec celui qu'a étudié Judith Walkowitz pour l'Angleterre
} de la fin du XIXe siècle (Walkowitz, 1992). 
modeste obligeant à se contenter de peu et des espoirs sentimentaux voire des ambitions de promotion sociale que leur démesure et leur irréalisme transforment en cauchemars. L'idée que les jeunes femmes sombrent dans la prostitution parce qu'elles n'ont pas voulu rester à leur place - au sens social comme géographique - est explicitement formulée par Philippon : "Au sortir de l'école où elles ne pouvaient rien apprendre, de la ruelle fangeuse d'une banlieue ouvrière où elles s'ennuient, elles ne trouvent rien de mieux à faire que d'arpenter les trottoirs des quartiers bourgeois pour y prendre l'argent et jouir du confort que la vie leur a refusé » (Philippon, 1954a, 25). Elle est également sensible, transposée dans une conception genrée du monde du travail, dans un article de Moissons nouvelles regrettant que trop de femmes entendent occuper des emplois masculins : "Lorsqu'elle veut ainsi singer l'homme, la femme en arrive à être obligée de travailler dans des conditions qui ne sont pas saines pour sa nature. (...) Prend-on assez soin de la délicatesse morale de la travailleuse, immergée ainsi durant des heures dans un climat masculin, avec une promiscuité souvent suspecte et au milieu de propos qui n'ont rien de respectueux pour les pudeurs de l'amour? Que de tentations, pour appeler les choses par leur nom, que de chocs pour le système nerveux » $\left(M S, \mathrm{n}^{\circ} 4,1952\right)$.

Un élément est fréquemment invoqué comme contribuant à cette vulnérabilité psychologique des futures prostituées: il s'agit des cultures et loisirs populaires, spécialement féminins. La presse du cœur est régulièrement dénoncée pour ce qu'elle prédisposerait les jeunes filles à croire les serments d'amour des plus vils séducteurs et, plus généralement, les inciterait à céder à leur fiancé avant le mariage, ce qui les exposerait à des grossesses non désirées $\left(M S, \mathrm{n}^{\circ} 6,1953\right)$. Les films de cinéma (et leurs affiches) sont l'objet de critiques récurrentes pour leur complaisance érotique et violente, ainsi que dans leur mise en scène favorable d'un monde "matérialiste et paganisé » $\left(M S, \mathrm{n}^{\circ} 19,1956,10\right)$ prédisposant à un esprit de "revanche du pauvre "subjugué par l'attrait du luxe et de l'argent" » $\left(M S, \mathrm{n}^{\circ} 10,1954\right)$. Le Cartel se montre sur ce point le plus véhément en demandant "aux pouvoirs publics et au Parquet, responsables du bien commun, de combattre le cinéma corrupteur, la littérature et l'affichage pornographiques, la multiplication exagérée des dancings et des bars louches, des boites de nuit et des concours de beauté » (Rénovation, $\mathrm{n}^{\circ} 11,1950$ ).

Que «le désir du bonheur" soit confondu «avec le plaisir dans un monde matérialisé » $\left(M S, \mathrm{n}^{\circ} 10,1954\right)$ trouve surtout son expression dans le domaine familial, considéré comme primordial par les abolitionnistes. Si tant de jeunes femmes se retrouvent sur le trottoir c'est, selon eux, parce qu'elles sont issues de familles « anormales » où règnent la promiscuité, l'alcoolisme et le manque d'amour. La première parmi les prédispositions à la prostitution est selon Philippon «la maternité hors mariage » (Philippon, 1954a, 24) qui expose à perdre son emploi ou à être chassée de son foyer. Le Nid affirme par conséquent que «tous ceux qui, d'une manière quelconque, nuisent à la formation et au maintien de familles normalement constituées, coopèrent pour une part à la prolongation de ce fléau. Nous croyons que les familles bâties selon le plan de Dieu sont la meilleure arme contre la prostitution » $\left(M S, \mathrm{n}^{\circ} 23,1957,6\right)$. En conséquence, le Nid s'oppose au divorce et regrette que les enfants conçus hors mariage puissent donner droit aux allocations familiales car cela « cela encourage l'union libre et ce que l'on pourrait appeler une polygamie de fait» (ibid., 59). Les abolitionnistes condamnent par ailleurs la contraception, l'avortement et l'homosexualité. À l'inverse, selon Philippon $(1958,83)$, «la continence sexuelle est, physiologiquement parlant, possible et inoffensive pour l'équilibre foncier de l'organisme vivant ». 
L'emprise de l'étiologie psychologico-familiale de la prostitution se mesure au fait qu'elle n'est pas seulement mobilisée pour rendre compte des conduites des prostituées, mais également de celles des souteneurs et des clients. S'agissant des premiers, le Nid indique qu'un grand nombre d'entre eux ont souffert d'une éducation «faussée ou inexistante dans une société où le divorce, le concubinage, les maternités hors mariage jettent dans l'existence sans forces morales et sans but une quantité d'enfants et de jeunes gens qui s'en vont inévitablement peupler les maisons de rééducation, les prisons et les rangs des souteneurs et des trafiquants » $\left(M S, \mathrm{n}^{\circ} 27,1959,48\right)$. Quant aux clients, c'est leur immaturité qui est soulignée: "Un homme de 40 ans qui a recours aux prostituées manifeste un état d'évolution de sa sexualité correspondant environ à 17 ou 18 ans » $\left(M S, \mathrm{n}^{\circ} 27,1959,56\right)$. Ces travers sont d'autant plus pernicieux qu'ils ont été contractés jeune, notamment $\mathrm{du}$ fait d'un accès facile et institutionnellement déculpabilisant aux maisons closes. En conséquence, le Nid préconise pour les adolescents et fiancés en recherche d'initiation « de modifier leur façon de concevoir la jeune fille et la femme trop souvent convoitées comme un objet de plaisir » (ibid.) tandis que le Cartel en appelle "à tous, étudiants ou jeunes ouvriers, soldats ou chefs de famille, de ne plus considérer la femme comme une bête de somme ou un instrument de plaisir » (Rénovation, $\mathrm{n}^{\circ} 11,1950$ ).

Les causes sociales de la prostitution ne sont pas pour autant négligées mais, on l'a dit, posées comme secondaires en regard des carences psychiques des jeunes femmes. L'ancrage du Nid dans le catholicisme social est au principe d'une sensibilité certaine à la condition ouvrière et il n'est pas anodin que nombre de ses équipières travaillent en usine pour - sur un modèle qui rappelle plus Simone Weil que les futurs établis maoïstes - partager la condition des classes laborieuses. Selon l'association, « le patron qui ne paie pas suffisamment son employée » et " l'industriel qui maintient dans son usine des conditions de travail inhumaines pour ses ouvrières" sont aussi responsables de «la prostitution de 600000 de nos compatriotes » que "l'avocat qui favorise le divorce » et le "médecin qui conseille et pratique l'avortement » $\left(M S, \mathrm{n}^{\circ} 2\right.$, 1952). Le Cartel n'est pas en reste qui demande "aux patrons de payer mieux leur personnel féminin et d'assurer le respect des jeunes filles de leurs entreprises» (Rénovation, $\mathrm{n}^{\circ} 11,1950$ ).

Partie prenante d'un conglomérat organisationnel où figurent également la JOC, la CFTC, le MPF ou encore la Confédération générale du logement, le Nid en relaie les revendications en matière sociale, familiale ou d'habitat. Estimant à $95 \%$ la part des prostituées "originaires du monde ouvrier», il loue par exemple l'initiative de celles qui, en 1963, organisent une quête de soutien pour des mineurs en grève $\left(M S, \mathrm{n}^{\circ} 51\right.$, 1964, 30). Cet ouvriérisme est toutefois ambivalent et la condition de travailleuse apparaît comme en elle-même lourde de périls pour les jeunes femmes qui s'emploient comme domestiques où à l'usine: "Tous les patrons qui emploient des jeunes filles venant de la campagne les mettent en danger, quand bien même tout serait parfait dans l'atelier ou le bureau » $\left(M S, n^{\circ} 4,1952\right)$. La condition ouvrière a beau être exaltée au sein du catholicisme social, son appréhension reste fondée sur une nette distinction nette entre les rôles masculin (de travailleur salarié) et féminin (d'entretien de la sphère domestique). De fait, c'est le foyer familial davantage que le monde du travail qui est posé en havre salvateur pour les prostituées en voie de reclassement.

Soulignons, pour clore cette section, que ses déclinaisons masculines sont absentes de la vision de la prostitution que développent alors les abolitionnistes. Celle-ci n'est envisagée qu'au féminin et si l'homosexualité masculine est mentionnée, c'est d'une manière qui unifie à la fois des pratiques vénales et gratuites, selon un modèle en 
continuité avec celui identifié pour le début du siècle par Régis Revenin (2005). Soulignons également combien la pratique de la prostitution est elle-même passée sous silence, spécialement dans sa dimension sexuelle: le dolorisme compassionnel des discours éclipse le fait que, concrètement, la prostitution consiste en des prestations sexuelles offertes contre rétribution à des clients. Pour pouvoir être publiquement évocable et pour devenir cause, la prostitution doit en quelque sorte être désexualisée.

\section{Le reclassement des prostituées}

$\mathrm{Si}$, on l'a vu, le mouvement abolitionniste ne revendique pas encore, sur la période considérée, l'abolition de la prostitution elle-même, il n'en entend pas moins lutter pour le "reclassement» de celles qui l'exercent. Le Cartel affirme en 1949 (Rénovation, $\mathrm{n}^{\circ} 5$ ) recevoir chaque semaine une moyenne de quinze prostituées «qui nous supplient de les sortir de leur triste métier » et lance un appel à " aider à rendre à une vie honnête et à la Famille les victimes d'une Société qui n'est plus à la taille de l'homme et qui est devenue, à cause de cette atmosphère viciée dans laquelle nous vivons, une monstrueuse machine à broyer les consciences ». Cet appel vise notamment les « industriels susceptibles de procurer un travail facile à réaliser dans notre petit atelier d'attente » (ibid.). Pour autant, cette activité de réinsertion paraît s'estomper au fil des années et n'est plus mentionnée par la suite dans la publication du Cartel. Comme indiqué plus haut, la création du Nid entraîne un partage des tâches entre associations abolitionnistes : au Cartel le travail de lobbying tandis que le Nid se spécialise dans la prise en charge à vocation sociale.

Le Nid est en premier lieu un foyer à destination des prostituées qui souhaitent abandonner leur activité. Celles-ci seraient une majorité et Moissons nouvelles avance que "si l'on s'aventurait à demander l'avis des "filles", $95 \%$ d'entre elles crieraient: "Nous ce que nous voulons c'est en sortir. Quitter ce métier d'esclaves" " (n 14-15, $1955,6)$. Pourtant, peu viennent d'elles-mêmes demander du secours et c'est une des tâches des équipiers que d'arpenter les trottoirs pour leur apporter un réconfort, faire connaître leur action et leur proposer de rejoindre le Nid. Cette démarche, outreach avant l'heure mais surtout application du modèle missionnaire, est ainsi décrite par Philippon : " [les équipiers] entrent volontiers au bar avec elles, leur offrent café ou jus de fruit, bavardent d'un sujet ou d'un autre. Ils renouvellent le plus souvent qu'ils peuvent ces visites d'amitié, dans l'espoir de surprendre chez la malheureuse un aveu de dégoût et de lassitude, pour lui tendre une main fraternelle, lui offrir la maison familiale d'accueil. Les repêchages se font surtout la nuit, entre 23 heures et 2 heures du matin » (Philippon, 1954b, 189).

L'entrée au foyer de celle qui s'est décidée à quitter le trottoir paraît ritualisée, selon des modalités - elle y est "attendue et accueillie par toute la famille et un paternel goûter» $\left(M S \mathrm{n}^{\circ} 1,1951\right)$ - qui évoquent l'intégration dans les institutions totales décrite par Goffman (1968). Et, de fait, le foyer du Nid prend bel et bien en charge la totalité de l'existence de la nouvelle entrante, qui y reste cloitrée pendant les premiers mois de sa prise en charge. Dotée d'une chambre individuelle, elle est amenée à participer aux cours et aux loisirs - français, hygiène et médecine, puériculture, cuisine, chant et musique, rythmique et culture physique, jardinage - « intensifs » (sans doute à fins de discipline et de prévention de l'oisiveté ${ }^{10}$ ) pendant les deux ou trois

\footnotetext{
${ }^{10}$ On ne peut manquer de penser ici à ce que dit Foucault de la lutte contre l'oisiveté dans Surveiller et punir (Foucault, 1975).
} 
premiers mois. Les pensionnaires travaillent également à l'atelier, ce qui leur permet de toucher un salaire destiné à se constituer un trousseau. Il s'agit d'instiller chez elles des dispositions au travail régulier que certaines ont perdues ou n'ont jamais acquises, et la tâche paraît parfois ardue même si le Nid estime que « les pousser à l'effort, c'est cela les aimer » $\left(M S, \mathrm{n}^{\circ} 20,1956\right)$. Il s'agit plus directement de les mettre en condition de se résigner à occuper des emplois pénibles et mal payés, seul sort professionnel envisageable pour des femmes dépourvues de qualification: «Le milieu de travail de demain sera dur, souvent inhumain et pourtant elles devront, comme tant d'autres, $y$ vivre, y peiner et y militer pour plus de justice et de dignité » $\left(M S, \mathrm{n}^{\circ} 16,1955\right)$. Des équipières font l'expérience douloureuse d'être flouées par des menteuses ou de se confronter à des paresseuses, et le foyer se révèle parfois incapable de prendre en charge des «malades mentales présentant des signes psychotiques, des grandes débiles et la plupart des homosexuelles » $\left(M S, \mathrm{n}^{\circ} 38,1961\right)$.

La carrière morale (Goffman, 1968) de l'ex-prostituée se poursuit par un séjour en famille d'accueil, choisie dans le vivier jociste, pour sa part destiné à leur faire découvrir un cadre familial positif propice à des relations humaines authentiques. Ainsi, " chaque fille passe (...) dans un foyer militant, ouvrier, rural ou bourgeois selon les cas, et, là, elle découvre le mariage, l'amour, la vie de famille, l'éducation des enfants, la vie de travail très dure des militants où, pourtant, on trouve tant de joie » $\left(M S, \mathrm{n}^{\circ} 16,1955\right)$. Le début d'une vie professionnelle nouvelle est ensuite soutenu par un hébergement de quelques semaines au foyer de travailleuses de Fontenay. Celui-ci offre en effet un encadrement destiné à parer les « tentations de jadis » et à épauler la travailleuse « dans ses difficultés, lui apprendre peu à peu à équilibrer un budget et à devenir une militante dans son travail » (ibid.). On ne saurait en effet sous-estimer la dimension politique du projet du catholicisme social qui, s'il se veut concurrent du socialisme, n'en est pas moins amené, de ce fait même, à intervenir sur les mêmes thèmes et terrains. Et ce sont bien des emplois ouvriers ou de service (relevant spécialement de ce qu'on désignerait aujourd'hui comme le care) faiblement qualifiés qui attendent les ex-prostituées. Le $\mathrm{n}^{\circ}$ 16 (1955) de $M S$ indique que les reclassements professionnels obtenus par le Nid se déclinent en quatorze en usine, huit employées de maison et aides familiales, une garde d'enfant, deux filles de salle dans des cliniques, une concierge, une femme de ménage, une serveuse de restaurant, un emploi de bureau, trois en formation (deux sténodactylos et une puéricultrice) et deux chômeuses.

Le terme de la carrière morale n'est pas seulement professionnel mais également familial. Il est attendu des anciennes prostituées qu'elles fondent à leur tour cette institution chérie entre toutes par les abolitionnistes qu'est la famille, et le Nid ne cesse de répéter que "les "filles" elles-mêmes, aimées, peuvent fonder de véritables foyers. Alors seulement les choses sont remises en ordre » $\left(M S, \mathrm{n}^{\circ} 23,1957\right)$. Moissons nouvelles regorge de récits édifiants de femmes sorties du Nid pour se marier (souvent avec un collègue de travail), devenir mères de famille et se muer en parfaites ménagères - on l'a $\mathrm{vu}$, leur salariat est davantage conçu comme un pis-aller temporaire que comme une condition durable. Le couple hétérosexuel apparaît ainsi comme la norme indépassable, conformément à la conception catholique de la complémentarité des sexes : «Personnes substantiellement achevées mais incomplètes, l'homme et la femme sont complémentaires l'un de l'autre. Incomplétude et complémentarité qu'on retrouve à tous les plans : physique (force de l'homme, fragilité de la femme), affectif (amour viril aux composantes charnelles fortement marquées, amour sentimental où la richesse du cœur a souvent la primauté), intellectuel (large conceptions et jugement solide, intuition des nuances et jugement pénétré d'émotivité) » $\left(M S, \mathrm{n}^{\circ} 23,1957, \mathrm{p} .37\right)$. 
L'achèvement de la carrière peut également prendre une forme spirituelle. Le parcours institutionnel au sein du Nid est, dans tous les cas, explicitement destiné à ouvrir "sur un ordre de valeurs autre que celui de l'argent et du plaisir » et il s'agit de favoriser l'«apparition ou ré-apparition progressive des tendances métaphysiques ou spirituelles qu'il faudra rendre "adultes". Et ici autant que le recours très libre au prêtre, la puissance d'amour de la communauté chrétienne dans laquelle baigne le Nid, marque profondément la fille et l'invite à faire, elle aussi, une expérience identique » (Séguier, 1954, 65). L'objectif, selon les termes de la même auteure, est de produire d' "autres "Madeleine" » en les ramenant dans le giron de la foi catholique (le Nid insiste régulièrement sur le fait qu'une majorité de prostituées sont des baptisées). La logique missionnaire d'évangélisation de celles et ceux qui méconnaissent le Christ, fondatrice du catholicisme social, se fait ici explicite. Elle s'appuie sur des dispositifs quotidiens rythmant la vie du foyer ou plus exceptionnels, comme ce voyage à Lourdes des pensionnaires organisé dans le cadre du " pèlerinage international du monde ouvrier » $\left(M S, \mathrm{n}^{\circ} 28,1958\right)$.

De sorte que la carrière atteint une sorte de couronnement lorsque le Nid parvient à reproduire ses propres effectifs à partir de la (re)conversion religieuse de son public, en d'autres termes quand d'anciennes prostituées deviennent jocistes, voire prennent le voile. Le travail en usine est dans certains textes lui-même présenté, plutôt que comme l'acceptation résignée d'un état du marché de l'emploi, comme le produit d'un désir missionnaire d'aider les autres salariées « à porter le poids de leurs rudes journées et leur faire connaître le secret du bonheur, le beau message d'amour qu'ellesmêmes, après tant de mois de souffrance et d'abandon, ont enfin connu " $\left(M S \mathrm{n}^{\circ} 1\right.$, 1951). Les entrées au couvent, pour leur part, ne sont pas toutes le produit d'une révélation spirituelle, comme lorsque « les plus diminuées » rejoignent une communauté de religieuses pour y finir leur vie $\left(M S, n^{\circ} 51,1964,32\right)$. Mais ce qui est avant tout valorisé sont les cas où « la grande détresse des "filles de joie" monte jusqu'au cœur de Dieu qui répond dans son amour en appelant des missionnaires », i.e. lorsque de nouvelles équipières entendent "participer de toutes [leurs] forces à cette action magnifique : l'évangélisation de la classe ouvrière » $\left(M S, \mathrm{n}^{\circ} 14-15,1955,41\right)$.

\section{Politiques de l'abolitionnisme}

Les pages qui précèdent auront permis de mesurer combien il est difficile de classer unilatéralement l'abolitionnisme comme un mouvement "progressiste » ou "conservateur». Manifestement puritain en matière de mœurs et promoteur d'une vision rigide des rapports entre les sexes, il témoigne également d'une forte sensibilité (certes plus affirmée au sein du Nid que du Cartel) aux inégalités économiques et sociales. Sa compassion à l'égard des prostituées est tout aussi ambivalente. Suscitée par les difficultés et souffrances que celles-ci endurent, elle n'en suppose pas moins une relation asymétrique, non exempte d'autoritarisme, dont témoignent les politiques promues au sein du mouvement.

\section{Progrès social et coercition morale}

On l'a signalé, l'abolitionnisme français ne peut dans les années 1950 se satisfaire de la loi du 13 avril 1946, trop partielle et dont il craint - à juste titre compte tenu des prises de positions répétées de parlementaires en faveur d'une "réouverture " qu'elle soit remise en cause. D'où l'insistance dans ses publications sur les tares du réglementarisme, dans des termes et avec des arguments qui restent globalement ceux 
d'avant-guerre : les maisons closes incitent à la débauche en la facilitant et en offrant de fausses garanties sanitaires, elles ne préviennent ni ne canalisent les perversions mais $\mathrm{au}$ contraire les encouragent, et leur réglementation administrative équivaut à une reconnaissance officielle d'une activité que les pouvoirs publics devraient au contraire combattre. En outre, leur interdiction n'empêche pas les établissements de prostitution de poursuivre leurs activités de manière semi-clandestine faute d'une action décidée de la police, pas plus que n'est entravée l'activité des proxénètes.

Les abolitionnistes s'indignent surtout du fichier médico-social instauré le 24 avril 1946. Pas plus que les anciennes formes de «mise en carte » celui-ci ne parvient à appréhender la totalité des personnes qui se livrent à la prostitution, et les rafles régulières de la police sont incapables d'identifier toutes les «insoumises». Pas davantage qu'auparavant, la surveillance sanitaire ne peut être efficace en ne ciblant que les prostituées : bien que formulée de manière neutre, la législation ne vise en pratique que les prostituées, jugées seules coupables de la propagation des infections vénériennes. Les abolitionnistes rejoignent à leur manière la labelling theory en s'inquiétant d'un étiquetage durable des femmes soumises au fichage: "Il serait souhaitable que quand une femme demande sa radiation du fichier sanitaire et que celle-ci est acceptée, elle ne soit soumise à aucune surveillance même par une Assistante sociale ! (...). En effet, pourquoi poursuivre une femme avec un passé dont elle veut se dégager?»(Dolceroca, 1954, 51).

Le mouvement milite en conséquence pour la suppression de tous les éléments réglementaristes résiduels, ainsi que pour une action policière efficace contre les proxénètes, trafiquants de femmes et autres tenanciers de maisons clandestines. Le Nid revendique plus spécifiquement un ensemble de mesures de nature sociale, visant à prévenir l'entrée de jeunes femmes sur le trottoir. Ces mesures (rassemblées dans $M S$, $\mathrm{n}^{\circ} 3$, 1952) sont pour certaines d'ordre général et s'inscrivent dans une démarche sociale globale, telle que pouvaient à l'époque la promouvoir les syndicats. L'accent est mis sur le manque d'opportunités professionnelles pour les femmes, les insuffisances des salaires féminins, les mauvaises conditions de travail et les carences de la formation professionnelle des milieux populaires (sont notamment revendiqués un allongement de la scolarité obligatoire et des écoles ménagères pour les « jeunes filles du peuple »). Une attention particulière est accordée à l'habitat: sont exigés des logements décents et suffisamment spacieux (permettant que «les garçons et les filles aient des chambres séparées »), la création de foyers de jeunes travailleurs et travailleuses pour les apprentis; ou encore la mise en place d'habitations familiales pour les «hommes déplacés» (dont, principalement, les Nord-Africains) «dont l'isolement favorise la prostitution ».

D'autres revendications sont plus spécifiques. Plusieurs tendent, de manière prévisible, à un renforcement et à un développement des activités menées par le Nid, par exemple «la création de centres d'apprentissage accéléré pour permettre aux femmes sans métier d'en apprendre un » et la multiplication des centres maternels pour les prostituées enceintes ou jeunes mères. D’autres exigent une égalité de traitement en matière vénérienne - égalité entre les sexes ("nous demandons pour les femmes le même régime que pour les hommes ») mais aussi entre pathologies ("nous demandons que les malades atteints de maladies vénériennes soient traités comme d'autres malades »). Sans doute revendication la plus innovante et radicale sur le plan à la fois politique et du genre, tant le Nid que le Cartel réclament « la suppression de la police des mœurs et la création (...) de services d'inspectrices sociales de police pour la protection physique et morale des femmes et des enfants » (Rénovation, $n^{\circ} 6,1949$ ). 
Mais un ensemble de revendications tranche par sa dimension coercitive, rappelant que pour présenter des aspects progressistes, l'abolitionnisme n'en est pas moins adepte des solutions autoritaires en matière de mœurs. Le vocabulaire de l'interdiction, de la condamnation et de la punition se déploie largement dans Rénovation et dans Moissons nouvelles pour exiger la sanction des " pères des enfants naturels », de " tout homme qui cherche dans la rue à entraîner une jeune fille ou femme dans la débauche », des cafés qui accueillent des consommateurs de moins de 18 ans et de ceux qui vendent «des boissons enivrantes aux moins de 21 ans des deux sexes » ainsi que de "tous les auteurs, imprimeurs et vendeurs de revues pornographiques et de films qui portent les jeunes gens vers l'amour érotique et la débauche » $\left(M S, \mathrm{n}^{\circ} 3\right.$, 1952). Le Cartel s'indigne dans un article au titre éloquent - «Pour la propreté de la rue. Un abcès qu'il faut crever » $\left(\mathrm{n}^{\circ} 14,1951\right)$ - que le racolage ne soit pas assez réprimé et met en cause une police corrompue. Le père Talvas promeut de son côté la création d'un délit de prostitution, "délit de simple police établi par des agents en uniforme » ou par «des assistantes de police en uniforme ». Destiné à prendre la relève du racolage, celui-ci permettrait de mettre un terme à l'« anomalie juridique qui veut qu'actuellement un agent de la paix n'ait pas le droit d'intervenir alors qu'il y a des femmes qui racolent ouvertement à côté de lui » $\left(M S, \mathrm{n}^{\circ} 14-15,1955,58\right)$. De fait, la compassion exprimée à l'égard des prostituées reste une relation foncièrement inégalitaire dont le paternalisme autorise l'utilisation de méthodes coercitives à l'égard de celles qu'il s'agit de secourir.

\section{Triomphe et délitement de la lutte contre la traite}

$\mathrm{Si}$ elles expriment des revendications similaires, les deux associations ne font toutefois pas preuve du même investissement dans le débat public. Le partage des tâches qui s'est instauré entre elles ne suffit pas à en rendre compte, et le poids de l'histoire doit également être convoqué. Héritier d'une croisade morale qui s'est consolidée dans la première moitié du siècle autour de la dénonciation de la traite des blanches, le Cartel est beaucoup plus sensible à une thématique que le Nid, nouveau venu dans cette cause, n'évoque que rarement. La dénonciation de la traite a toujours été étroitement liée à celle du réglementarisme dans la rhétorique des abolitionnistes, selon lesquels le renouvellement du personnel des maisons closes appellerait le commerce international des femmes. C'est sans doute pourquoi les affaires de traite dénoncées dans Rénovation concernent essentiellement les colonies d'Afrique du Nord, où la loi de 1946 ne s'applique pas mais où, aux dires de J. Scelles, il conviendrait de répondre aux exigences de réforme des mœurs de la population musulmane par une " élévation morale de la jeunesse » et une répression accrue du proxénétisme $\left(\mathrm{n}^{\circ} 15-16\right.$, 1951-1952).

Le thème de la traite est surtout prétexte à stigmatiser le refus du gouvernement français de signer la Convention pour la répression de la traite des êtres humains et de l'exploitation de la prostitution d'autrui, dont le Cartel salue fin 1949 l'adoption par l'ONU tout en regrettant que la presse hexagonale ait ignoré l'événement. Rénovation s'indigne que la France et la Grande-Bretagne aient voté contre « l'abolition de la plus dégradante des servitudes imposées à la femme » $\left(n^{\circ} 9,1950\right)$ et se demande si le " pays des droits de l'homme» acceptera encore longtemps "d'être le dernier bastion de l'esclavagisme dans le monde " $\left(\mathrm{n}^{\circ} 22,1954\right)$. Profitant des élections législatives de l'hiver 1956, le Cartel propose aux candidats de rallier son "programme d'action positive contre les fléaux sociaux », parmi lesquels la traite des femmes et l'exploitation 
commerciale de la prostitution d'autrui, et leur demande de s'engager, en cas d'élection, à obtenir la ratification de la Convention $\left(\mathrm{n}^{\circ} 30,1956\right)$.

Centrale dans la presse du Cartel, l'exigence de ratification de la Convention de l'ONU est pratiquement absente de celle du Nid. C'est seulement en 1959 qu'apparaît pour la première fois dans Moissons nouvelles ( $\mathrm{n}^{\circ} 29$ ) l'exigence d'une ratification "sans tarder » de ce qui est présenté comme « la convention de 1949 contre la traite des êtres humains ». Cette appellation incomplète traduit peut-être la distance entre l'action alors menée par le Nid auprès des prostituées et la manière dont les institutions internationales ont mis en forme le thème de la traite. Ce dernier est certes présent dans son discours, on l'a vu spécialement au travers de la figure idéale de la victime naïve de vils souteneurs, mais il apparaît surtout comme un arrière-plan constitutif de l'identité abolitionniste du mouvement. Les prostituées que le Nid accueille dans ses foyers ne sont pas des victimes de la traite telle que leur image s'est constituée à la fin du XIX siècle et consolidée dans les conventions internationales successives (Chaumont, 2009). Certes elles aussi définies par une incorrigible crédulité, ces femmes n'ont pas migré de pays en pays sous la contrainte mais ont d'elles-mêmes quitté leur région d'origine tout en restant à l'intérieur des frontières de l'hexagone, et leurs souteneurs ne sont pas décrits comme des trafiquants internationaux organisés. De sorte que le tournant des années 1950 et 1960 assiste à la fois à la consécration institutionnelle de la lutte contre la traite et à son effacement de la manière dont les acteurs de terrain - associatifs mais également policiers - appréhendent la réalité prostitutionnelle ${ }^{11}$.

Cet effacement explique peut-être que Rénovation ait accordé un écho enthousiaste mais somme toute limité à la ratification de la Convention de l'ONU par la France via le vote de l'ordonnance du 28 juillet 1960 ( $\left.\mathrm{n}^{\circ} 42,1960\right)$. De fait, la prostitution s'est depuis quelques années estompée - et s'estompera plus encore les années suivantes - des pages du trimestriel du Cartel, au profit d'une dénonciation des dangers de la télévision, du twist, de la contraception, de la drogue et des "grands ensembles» propices aux «inadaptations juvéniles» $\left(n^{\circ} 47,1962\right)$. Tout se passe comme si, anticipant la victoire finale de son long combat pour l'abolition complète de la réglementation de la prostitution, le courant historique de l'abolitionnisme avait progressivement délaissé le terrain prostitutionnel pour privilégier la résistance aux évolutions en cours des mœurs et des cultures populaires. Ce faisant, il a laissé le champ libre à une mouvance catholique spécialisée dans le traitement social de la prostitution, à laquelle le nouveau contexte législatif va offrir les moyens de prospérer.

De ce point de vue, l'ordonnance qui suit de deux jours celle autorisant la France à ratifier la convention de l'ONU ${ }^{12}$ est plus décisive pour le mouvement abolitionniste

\footnotetext{
${ }^{11}$ L'année où la députée MRP Francine Lefebvre déclare, en se fondant sur les chiffres du service de recherche dans l'intérêt des familles que 100000 Françaises ont été enlevées pour la traite des blanches (propos repris dans $M S \mathrm{n}^{\circ} 18,1956$ ainsi que, malgré quelques réserves, dans Rénovation $\mathrm{n}^{\circ}$ 31, 1956), un rapport policier signale que les enquêtes pour traite "n'ont en général abouti qu'à établir, soit l'inexistence du "cas" signalé, soit une fugue volontaire n'ayant aucun rapport avec une affaire de proxénétisme »; cité in Mainsant (2012, p. 105). Les Équipes d'action contre la traite des femmes et des enfants abandonneront quant à elles toute référence à la traite au début des années 1990 en devenant Équipes d'action contre le proxénétisme. Ce n'est qu'au début des années 2000 que le thème de la traite retrouvera une place centrale dans le discours abolitionniste (Mathieu, 2014).

${ }^{12}$ L'ordonnance du 30 juillet 1960 met en place un ensemble de mesures destinées à lutter contre certains fléaux sociaux dont, outre la prostitution, l'alcoolisme, la tuberculose et, par un amendement de dernière minute, l'homosexualité. Les débats parlementaires qui l'ont précédée ont peu abordé la prostitution et se sont surtout concentrés sur l'alcoolisme, les députés des circonscriptions viticoles ayant activement résisté à son adoption; Journal officiel, compte rendu intégral des débats à l'Assemblée nationale, $2^{\mathrm{e}}$ séance du 18 juillet 1960, 19 juillet 1960.
} 
car elle marque l'abandon de la définition de la prostitution comme problème sanitaire pour la reconnaître comme problème social. Elle instaure plus précisément dans le Code de la famille et de l'aide sociale de nouvelles dispositions concernant l'assistance aux personnes prostituées ou en danger de prostitution. Déjà prévus par une ordonnance de 1958, le dépistage et la prise en charge des mineurs en danger de prostitution sont étendus aux majeurs et confiés à un service également chargé d'exercer toute action médico-sociale (placement en établissement, fourniture d'un emploi, orientation vers les services d'hygiène sociale ou de réadaptation, etc.) en faveur des personnes qui se livrent à la prostitution. Prévus pour l'ensemble de la France et pensés comme relevant de l'aide sociale préfectorale, ces services ne verront en réalité le jour que dans une dizaine de départements. Outre que le problème de la prostitution ne se pose pas avec la même acuité sur l'ensemble du territoire, les préfets préféreront souvent confier au secteur privé, et spécialement au Nid qui a su s'imposer au cours de la décennie précédente comme une référence en la matière, la tâche de développer cette assistance spécialisée ${ }^{13}$.

\section{Conclusion}

La quinzaine d'années qui sépare les lois d'avril 1946 des ordonnances de 1960 n'assiste pas seulement à l'ultime mobilisation préludant l'abandon complet du réglementarisme par la France. Le triomphe de l'abolitionnisme intervient paradoxalement au moment où s'épuisent une conception du problème prostitutionnel - celle qui, depuis la fin du XIX ${ }^{\mathrm{e}}$ siècle, associe traite et réglementation - et les forces militantes qui la portaient, cela au profit d'une approche à dominante sociale valorisée par une mouvance nouvelle issue du catholicisme social, celle du Nid. En s'investissant dans la cause de femmes stigmatisées perçues comme autant d'incarnations du Christ et en développant pour ce faire une approche spécialisée centrée sur la réinsertion, ces catholiques ont, sur le terrain de la prostitution, évincé ceux qui dominaient l'abolitionnisme depuis ses origines, à savoir les protestants.

Cette brève séquence historique offre un contrepoint comparatif avec la situation contemporaine. Le mouvement abolitionniste n'a certes pas disparu après avoir remporté son triomphe de 1960. Sa promotion d'une approche sociale de la prostitution a permis le développement d'un secteur spécialisé du travail social, au sein duquel l'Amicale du Nid, issue en 1971 de la scission avec le Mouvement du Nid, occupe aujourd'hui une position centrale. Longtemps cantonné à une posture défensive devant ce qui menace ses acquis (les propositions de réouverture des maisons closes et, de manière plus sérieuse, le retour d'une approche sanitaire imposée par la lutte contre le sida), il a repris l'offensive à partir de la fin des années 1990 à la faveur d'une alliance inédite avec le mouvement des femmes et d'une redéfinition de ses buts et de son identité : l'abolition qu'il vise n'est plus celle de la réglementation mais celle de la prostitution elle-même, dénoncée comme une violence sexiste redevable d'une

\footnotetext{
13 Le nouveau dispositif légal est complété quelques mois plus tard par deux ordonnances du 25 novembre 1960 qui en tirent les conséquences du point de vue de la lutte contre les maladies vénériennes (désormais menée avec les mêmes moyens et avec les mêmes obligations pour les prostituées comme pour le reste de la population) et de la répression du proxénétisme (en appelant à une répression redoublée contre les établissements de prostitution ou hôtels de passe ainsi qu'à des peines d'emprisonnement sans sursis contre toute " organisation de proxénétisme »). Le même texte prévoit une aggravation des peines pour racolage tout en affirmant respecter la «liberté de l'exercice de la prostitution » pour « celui qui s'y livrerait individuellement » (circulaire du 25 novembre 1960 relative à la répression du proxénétisme, publiée au Journal officiel du 27 novembre 1960).
} 
démarche avant tout pénale (Mathieu 2014). De fait, les évolutions récentes de l'abolitionnisme paraissent beaucoup plus importantes que celles qu'il a connues entre 1946 et 1960. Persiste cependant, en dépit de ces recompositions, ce qui apparaît comme un trait structurant du mouvement, à savoir une approche paternaliste (Donegani 2011) dont la dimension compassionnelle contribue autant à disqualifier la parole des premières concernées qu'à légitimer une gestion autoritaire de leur condition.

\section{Bibliographie}

BECKER H.S., 1985, Outsiders, Études de sociologie de la déviance, Paris, Métailié.

BLANCHARD V., 2008, Les Filles «perdues» sont-elles amendables? Les mineures prostituées devant le tribunal pour enfants de la Seine dans les années 1950, Revue d'histoire de l'enfance "irrégulière », 10, 35-55.

BLAND L., 1992, "Purifying " the public world: Feminist vigilantes in late Victorian England, Women's History Review, 1, 3, 397-412.

BOLTANSKI L., 1993, La Souffrance à distance, Paris, Métailié.

BRUNET J.-B., 1990, Évolution de la législation française sur les maladies sexuellement transmissibles, in JOB-SPIRA N., SPENCER B., MOATTI J.-P., BOUVET E. (dir.), Santé publique et maladies à transmission sexuelle, Paris, John Libbey Eurotext, 113-121.

CHAUMONT J.-M., 2009, Le Mythe de la traite des blanches. Enquête sur la fabrication d'un fléau, Paris, La Découverte

CHAUMONT J.-M., MACHIELS C. (dir.), 2009, Du sordide au mythe. L'affaire de la traite des blanches (Bruxelles, 1880), Louvain, Presses universitaires de Louvain.

CORBIN A., 1978, Les Filles de noce, Paris, Aubier.

DOLCEROCA L., 1954, La prostituée est aussi une personne, Cahiers Recherches et débats, 9, 40-55.

DONEGANI Jean-Marie, Le paternalisme, maladie sénile du libéralisme?, Raisons politiques, 44, 5-14.

DUFFULER-VIALLE H., 2014, Le «Chant du cygne». De la prostitution réglementée durant l'entre-deux guerre, communication à la journée d'étude "La Prostitution urbaine en Europe du Moyen-âge à nos jours », Université Toulouse Jean-Jaurès.

FOUCAULT M., 1975, Surveiller et punir, Paris, Gallimard.

GEMÄHLING P., 1933, La Réglementation administrative de la prostitution jugée d'après les faits, Bordeaux, éditions du Relèvement social.

GOFFMAN E., 1968, Asiles. Études sur la condition sociale des malades mentaux, Paris, Minuit.

JAKSIC M., 2013, Devenir victime de la traite. L'épreuve des regards institutionnels, Actes de la recherche en sciences sociales, $\mathrm{n}^{\circ} 198$, p. 36-49.

KÄPPELI A.-M., 1990, Sublime croisade. Éthique et politique du féminisme protestant 1875-1928, Genève, Zoé 
LASSERRE J., 1955, Comment les «maisons » furent fermées, Genève, Fédération abolitionniste internationale.

LIMONCELLI S.A., 2010, The Politics of Trafficking. The First International Movement to Combat the Sexual Exploitation of Women, Stanford, Stanford University Press.

MAINSANT G., 2012, L'État et les illégalismes sexuels. Ethnographie et sociohistoire du contrôle policier de la prostitution à Paris, thèse de sociologie, EHESS.

MATHIEU L., 2005, Repères pour une sociologie des croisades morales, Déviance et société, $29,1,3-12$.

MATHIEU L., 2014, La Fin du tapin. Sociologie de la croisade pour l'abolition de la prostitution, Paris, François Bourin.

MAUGÈRE Amélie, 2009, Les Politiques de la prostitution du Moyen Âge au XXIe siècle, Paris, Dalloz.

OLIVIER C., 2005, Le Vice ou la vertu. Vichy et les politiques de la sexualité, Toulouse, Presses universitaires du Mirail.

PELLETIER D., 2012, Une Gauche sans domicile fixe, in Pelletier D., Schlegel J.-L. (dir.), $\grave{A}$ la gauche du Christ. Les chrétiens de gauche en France de 1945 à nos jours, Paris, Seuil, 1751 .

PHILIPPON O., 1954a, Connaissance de la prostitution, Cahiers Recherches et débats, 9, 930.

PHILIPPON 0., 1954b, L'Esclavage de la femme dans le monde contemporain ou la prostitution sans masque, Paris, Téqui.

PHILIPPON 0., 1958, Un Grave danger pour la jeunesse du monde: la traite des êtres humains, Paris, Téqui.

REGARD F. (dir.), 2013, Féminisme et prostitution dans l'Angleterre du XIXe siècle : la croisade de Josephine Butler, Lyon, ENS éditions.

REVENIN R., 2005, Homosexualité et prostitution masculines à Paris, Paris, L'Harmattan.

ROCHEFORT F., 2008, The Abolitionist Struggle of Pastor Tommy Fallot: Between Social Christianity, Feminism and Secularism (1882-1893), Women's History Review, 17, 2, 179-194.

SACOTTE M., 1959, La Prostitution, Paris, Correa.

SEGUIER M.-J., 1954, Comment sauver? L'expérience du Nid, Cahiers Recherches et débats, 9, 56-68.

TARAUD C., 2003, La Prostitution coloniale. Algérie, Tunisie, Maroc (1830-1962), Paris, Payot.

TRAÏNI C (dir.), 2009, Émotions... Mobilisation !, Paris, Presses de Sciences-Po.

WALKOWITZ J.R. 1980, Prostitution and Victorian Society, Cambridge, Cambridge University Press.

WALKOWITZ J.R., 1992, City of Dreadful Delight, Londres, Virago. 